\title{
Desempenho Acadêmico E Uso De Tecnologias De Informação E Comunicação: Uma Análise Com Estudantes De Graduação Do Nordeste Brasileiro
}

\section{Academic Performance And The Use Of Information And Communication Technologies: An Analysis With Undergraduate Students From Brazilian Northeast}

\author{
Ademir Macedo Nascimento ${ }^{1}$, Jananda da Silva Pinto ${ }^{2}$, Adauto José Valentim Neto ${ }^{2}$
}

\author{
${ }^{1}$ Universidade de Pernambuco, UPE, Brasil \\ ${ }^{2}$ Universidade Federal de Pernambuco, UFPE, Brasil \\ Correspondência: Ademir Macedo Nascimento. Endereço: Rua Amaro Maltês de Farias, Nazaré da Mata - PE, \\ 55800-000 Telefone: (81) 3633-4600 E-mail: ademir.nascimento@upe.br
}

Recebido: 30 de novembro de 2017 Aceito: 15 de dezembro de 2017 Publicado: 31 de dezembro de 2017

DOI: http://dx.doi.org/10.21714/1679-18272017v15Ed.p138-146

\begin{abstract}
Resumo
A prática lúdica de jogar é realizada desde tempos primórdios como forma de aprimoramento intelectual dos indivíduos. Neste cenário, os jogos podem atuar como dispositivos culturais que propiciam diversão, alegria, descontração, bem-estar ou como, em conotação ontológica, uma execução básica da vida. Neste cenário, é cada vez mais notável a difusão de jogos por meio das tecnologias de informação, além de uma série de outros usos da TI como relacionamento social, ativismo, relacionamento com empresas, busca de informações, entre outros. A partir desta gama de oportunidades, este estudo tem como proposição discutir as relações entre os diversos usos de tecnologia da informação e sua relação com as escolhas de graduandos, seja com relação ao que os levou a escolher o curso ou com relação a suas intenções de carreira ao se formar.
\end{abstract}

Palavras-chave: Tecnologia da informação. Graduação. Carreira.

\begin{abstract}
The playful practice of playing has been carried out since the earliest times as a way of intellectual improvement of individuals. In this scenario, games can act as cultural devices that provide fun, joy, relaxation, well-being or as, ontological connotation, a basic execution of life. In this scenario, it is increasingly noticeable the diffusion of games through information technologies, in addition to a series of other IT uses such as social relationship, activism, relationship with companies, information search, among others. From this range of opportunities, this study has as a proposition to discuss the relations between the various uses of information technology and its relationship with the choices of undergraduates, whether in relation to what led them to choose the course or in relation to their intentions of career when graduating.
\end{abstract}

Keywords: Information technology. University graduate. Career.

Esta obra está licenciada sob uma Licença Creative Commons Attribution 3.0.

\section{Introdução}

$\mathrm{Na}$ educação de crianças e jovens, práticas lúdicas permitem o aprimoramento intelectual dos indivíduos por meio de dispositivos culturais que propiciam diversão, alegria, descontração, bem-estar ou como, em conotação ontológica, uma execução básica da vida (SILVA, 2008).

Silva (2008, p. 16) descreve ainda o envolvimento do indivíduo com tal realidade lúdica e sua dinâmica da seguinte forma: 
Na medida em que faz imergir o ser humano em sua dinâmica, envolve-o afetivamente fazendo-o desenvolver planos de ação, modelos, procedimentos, estratégias, para obtenção de determinados fins. Neste sentido, jogar é, então, buscar, aprender, envolver-se, engajar-se na própria vida e ir em busca da felicidade. E como o ser humano não é uma ilha, toda essa dinâmica acontece em sociedade.

Neste cenário, é cada vez mais notável a difusão de jogos como dispositivos lúdicos, em especial aqueles disponíveis em plataformas eletrônicas, que permitem, além do uso para fins educacionais, uma série de usos como relacionamento social, ativismo, relacionamento com empresas, busca de informações, entre outros (NASCIMENTO; LUFT; SILVEIRA, 2014).

A partir desta gama de oportunidades, este estudo tem como proposição analisar as relações entre uso de tecnologias de informação e comunicação e jogos nas escolhas e no desempenho dos estudantes de cursos de graduação de universidades da região Nordeste do Brasil, uma vez que a teoria da mediação cognitiva ressalta melhorias de desempenho e de empreendedorismo em estudantes com alto grau de uso de tecnologias de informação.

Este estudo teve como intenção analisar as relações entre uso de tecnologias de informação e comunicação e jogos nas escolhas e no desempenho dos estudantes de cursos de graduação de universidades da região Nordeste do Brasil, uma vez que a teoria da mediação cognitiva ressalta melhorias de desempenho e de empreendedorismo em estudantes com alto grau de uso de tecnologias de informação.

Para tanto, este artigo está estruturado em seis seções a partir desta, tratando do uso de tecnologias de informação na educação e de jogos na educação nas duas seções seguintes, os procedimentos metodológicos, a discussão dos resultados logo após e, por fim, as conclusões.

\section{Uso de tecnologias da informação e sua relação com a educação}

O uso da tecnologia da informação é cada vez mais crescente entre a população, principalmente no que se refere à tecnologia mobile (VAFA; CHICO, 2013), o que torna inevitável que os ambientes educacionais se aproximem dessa realidade. Como argumenta Selwyn (2016), o próprio aumento do uso da tecnologia em outros lugares da sociedade evoca uma mudança na educação para que se adapte ao uso da tecnologia dentro dos ambientes educacionais ou nas situações de aprendizado.

Não obstante, a educação nos dias de hoje não pode ser distanciada da tecnologia. Sendo assim, existe um interesse generalizado de empresas e do governo nas formas como a tecnologia pode ser integrada na educação de forma benéfica (HEROLD, 2016).

Herold (2013) destaca que tal interesse pode ser visto de várias maneiras, a citar:

- A formulação de tecnologia específica em políticas educacionais em muitos países do mundo;

- Na alocação de importantes recursos econômicos e esforços no investimento humano;

- No crescente número de oportunidades colaborativas de troca de informações, conhecimentos e experiências;

- Nas muitas iniciativas educativas inovadoras lideradas pelo profissionalismo dos professores.

Essa última se justifica pela possibilidade de como os seres humanos aprendem, seja por inferência, observação ou associação, ao passo que a aprendizagem é desenvolvida também por meio de experiências, regras, iniciativas, métodos e estratégias de ação, ou ainda por meio da criação de lógicas adaptadas às necessidades de resolução de problemas para sobrevivência (BANDURA, 2000; LUSZCZYNSKA; SCHWARZER, 2005; CARNEIRO et al., 2013). Tais lógicas, quando em crianças, surgem a partir de cenários lúdicos no qual elas se imergem em um contexto com regras e significados culturalmente partilhados e envolvidos afetivamente (SILVA, 2008).

Um dos tipos de jogos lúdicos mais famosos na atualidade, principalmente entre jovens e adolescentes, são os do gênero RPG (Role Playing Game). Esse tipo de jogo faz jus ao contexto de interpretação de personagens, em cenários de enredos imaginários, por mediação de um mestre do jogo (game master) que narra a história na qual estão inseridos os jogadores (BITTENCOURT; GIRAFFA, 2003).

Randi e Carvalho (2013) realizaram uma pesquisa com alunos do curso de medicina submetidos a aulas expositivas tradicionais e a aulas com RPG concluindo que a aceitação do uso do jogo nas aulas se justifica porque o RPG incentiva a cooperação e a participação ativa dos alunos. Ao contar uma história em que todos participam, há uma maior interação dos alunos e, como consequência, espera-se um melhor desempenho na 
construção do conhecimento.

Os autores ainda complementam que o RPG pode prosseguir em várias direções, vez que oferece aos estudantes diferentes possibilidades para consolidar o novo conhecimento aprendido, ao contrário do que acontece, usualmente, nas aulas expositivas tradicionais (RANDI; CARVALHO, 2013).

Ademais, Pereira e Dalmau (2015) e Prazeres (2015) destacam a importância do desenvolvimento de competências virtuais no ensino superior, ressaltando um universo simbólico de valores relacionados aos aparatos digitais que formam uma ambiência favorável às tecnologias no campo educacional.

$\mathrm{Na}$ Era digital, o surgimento dos computadores e as telecomunicações, mudaram de forma geral a visão dos jogos considerados de interpretação, como os RPGs. Além disso, Silva (2008) acredita que essa evolução introduziu quatro importantes capacidades aos dispositivos, como:

- Automação repetitiva de mecânica de jogo, lançamento de dados, posicionamento de personagens, cálculos, manutenção de registros etc.;

- Representações audiovisuais ricas e sincronizadas com os eventos no jogo, com animações, filmes, sons, voz, figuras e música;

- Complexa interação de mundos virtuais gerada por inteligência artificial, ambientes que influenciam e são influenciados de acordo com as propriedades dinâmicas e regras predefinidas;

- Interações de longa distância tanto entre jogadores como no próprio jogo.

Ainda para Silva (2008), cada tipo de jogo detém de um funcionamento dinâmico e social distinto e próprio. Os jogos solitários e os de rede em suas relações tendem a ser limitados e breves, sendo que no primeiro existe a possibilidade de formação de grupos em torno dele, mas a interação direta não existe, diferente do segundo tipo de jogo onde essa interação existirá.

Por outro lado, os MMORPGs proporcionam uma interação social de longo prazo, direcionadas à utilização de estratégias mais ricas em detalhes e mais complexas, possibilitando diferentemente dos outros jogos (solitários e de rede) a interação competitiva direta entre os jogadores. Os MMORPGs podem contribuir para o desenvolvimento das habilidades sociais segundo os estudos de Ducheneaut e Moore (2005), tanto nas habilidades de cooperação como na de coordenação de atividades, o jogo permite três tipos distintos de interação social: auto-organização dos jogadores, coordenação instrumental e downtime sociability.

Silva (2008) coloca que Steinkuehler e Chmiel (2006), fundamentados em dados coletados de discussões provenientes de fóruns online, discutem sobre como o pensamento científico é fomentado por MMORPGs, especificamente o World of Warcraft, em virtude da lógica e interações sociais inerentes ao seu ambiente virtual interno e realidade externa. Silva $(2008$, p. 61) traz que os autores afirmam ser possível classificar três contribuições cognitivas relativas aos usuários estudados:

1. Práticas Discursivas Científicas: dizem respeito à construção de conhecimento colaborativo, construído sobre as ideias dos outros, e de contra-argumentos que são característicos dos "hábitos mentais científicos";

2. Raciocínio Baseado-em-Modelo: construções mentais formadas por sistemas de entendimento, mecanismos de feedback entre seus componentes, e a utilização de descrição matemática das relações entre tais componentes;

3. Compreensão Teoria-Evidência: embora mais rara entre os indivíduos do grupo pesquisado, aparece como um entendimento da função e da relação entre teorias e evidências empíricas, o que também se reflete na capacidade de generalizar conhecimentos adquiridos em pequena escala, por assim dizer.

\section{Procedimentos Metodológicos}

O presente estudo teve como intenção realizar uma análise exploratório-descritiva sobre as relações entre os diversos usos de tecnologia de informação e o desempenho acadêmico de estudantes universitários da região do nordeste brasileiro.

Para realizar este objetivo, o estudo se posicionou como quantitativo e de corte transversal, uma vez que pretendeu medir as correlações entre as variáveis citadas, mas fazendo isso em apenas um dado momento (COOPER, SCHINDLER; 2011).

Para isso, utilizou-se estratégia de pesquisa de survey, para qual foi elaborado um questionário virtual que foi disseminado em grupos de universidades da região nordeste do Brasil, contando com 535 respostas válidas, 
representando todos os nove estados da região, a saber: Maranhão, Piauí, Fortaleza, Rio Grande do Norte, Paraíba, Pernambuco, Alagoas, Sergipe e Bahia. O questionário foi distribuído entre os meses de setembro a dezembro de 2016 por meio de um link para acesso e respostas on-line e divulgado em grupos de redes sociais compostas por universidades dos estados selecionados.

Após a coleta de dados, foi realizada inicialmente a estatística descritiva e posteriormente foram realizados os testes inferenciais, sendo o primeiro o teste de correlação de Spearman entre variáveis e posteriormente a Anova de Kruskal-Wallis, uma vez que nenhuma das variáveis se mostrou gaussiana (HAIR et al. 2009).

\section{Análise dos Resultados}

Nesta seção serão apresentados os resultados do estudo, no qual inicialmente se fará uma análise descritiva do estudo e posteriormente serão demonstradas as análises de correlação e Anova.

\subsection{ANÁLISE DESCRITIVA}

Ao total, foram pesquisados 535 estudantes de graduação de universidades localizadas no nordeste do Brasil. A idade dos respondentes variou de 17 a 57 anos, com média de 23 anos. No que se refere à renda familiar, 156 entrevistados afirmaram que os rendimentos familiares concentram-se entre dois e quatro mil reais mensais.

Já no que tange à ocupação dos entrevistados, 358 afirmaram que são exclusivamente estudantes enquanto 99 dos participantes afirmaram ser estagiários com média de 12 horas de trabalho semanais, ao passo que a média de horas de estudo fora da sala de aula foi de 11 horas semanais.

Com relação à área do curso, a maioria dos entrevistados era da área de ciências sociais aplicadas, seguido de estudantes de engenharias, ciências da saúde e ciências exatas, além de serem, em sua maioria, estudantes de universidades públicas. A distribuição em relação ao período do aluno se mostrou bastante homogênea, com alunos com pouco e muito tempo de curso, sendo que a maior parte destes alunos avaliou o curso como bom ou regular.

Dentre os itens que caracterizavam uma boa faculdade, os respondentes destacaram o nível dos professores e o conteúdo prático, sendo que o item menos importante foi a reputação da instituição.

Em relação as escolhas para a carreira, a maioria dos respondentes planeja trabalhar em órgãos públicos ou seguir carreira acadêmica, e pretende também cursar uma pós-graduação, uma vez que a enxerga como a melhor forma de melhorar sua qualificação assim que terminar o curso. Além disso, a maioria dos respondentes informou que escolheu o curso atual por ter simpatia pela profissão e se sentir apto para exercê-la, sendo que o item menos importante neste quesito foi a vontade dos familiares.

Nos itens relacionados ao uso de computadores, destaca-se que a maioria dos respondentes usa computador regularmente há cerca de nove anos, passando em média 24 horas utilizando-o por semana, enquanto utiliza o smartphone em média 33 horas por semana.

Ao utilizar o computador, o uso de e-mail e a busca de informações foram os itens mais destacados, seguidos de bate-papos e editores de texto. No outro oposto, utilizar sistemas de gestão e tratamento de imagens foram os usos menos destacados.

Já em relação ao uso para jogos, nota-se que a média de horas jogando semanalmente foi de 7 horas e a avaliação dos jogos, destacou os jogos de Quiz e de RPG, ao passo que se demonstrou-se menor interesse por jogos de esportes e de quebra-cabeças.

No último item, ao se auto avaliarem, os respondentes demonstraram uma melhor qualificação nos quesitos inteligência e relacionamento interpessoal, ao passo que apontam valores menores no que tange à realização profissional e a falar em público.

\subsection{ANÁLISE INFERENCIAL}

Nesta seção serão apresentadas as análises inferenciais, iniciando pelas análises de correlações feitas entre variáveis e posteriormente por análise de Anova para observar diferenças estatisticamente significativas entre grupos.

\subsubsection{Análise de correlações}

Após a análise descritiva, buscou-se aqui identificar correlações estatisticamente significativas entre as variáveis estudadas. Para tal, foi utilizada a correlação de Spearman, uma vez que não se tratavam de variáveis gaussianas.

Inicialmente observaram-se as horas semanais de trabalho e os usos de computadores, no qual foi possível identificar correlações positivas com uso de planilhas eletrônicas e uso de e-mail. Já em relação às horas 
semanais de estudo, foi destacada a correlação positiva com uso de editor de textos, apresentações e busca de informações. Tais relações se mostram bastante coerentes com as atividades desempenhadas, revelando o tipo de uso de tecnologia mais apropriado em casa caso. Na tabela 1, é possível observar tais correlações.

Tabela 1: Correlações com horas semanais

\begin{tabular}{cccccc}
\hline Tipo de atividade & $\begin{array}{c}\text { Planilhas } \\
\text { Eletrônicas }\end{array}$ & $\begin{array}{c}\text { Uso de } \\
\text { e-mail }\end{array}$ & $\begin{array}{c}\text { Uso de editor } \\
\text { de textos }\end{array}$ & $\begin{array}{c}\text { Uso de } \\
\text { apresentações }\end{array}$ & $\begin{array}{c}\text { Busca de } \\
\text { informações }\end{array}$ \\
\hline $\begin{array}{c}\text { Horas Semanais de } \\
\text { Trabalho }\end{array}$ & $0,16^{*}$ & $0,09^{*}$ & & & \\
$\begin{array}{c}\text { Horas semanais de } \\
\text { estudo }\end{array}$ & & & $0,12^{*}$ & $0,10^{*}$ & $0,12^{*}$ \\
\hline
\end{tabular}

Fonte: dados da pesquisa (2017)

Curiosamente, o nível de renda não demonstrou correlação com nenhum tipo de uso de computadores, mas o tempo de uso de computadores em anos teve correlação com planilhas eletrônicas $(0,08)$, o que pode se dever ao fato de usuários mais antigos estarem acostumados a este tipo de software.

A quantidade de horas usando smartphone teve correlação positiva com bate-papo $(0,16)$, o que provavelmente reflete a ascensão de aplicativos como o WhatsApp ${ }^{\circledR}$, Messenger $^{\circledR}$, Viber $^{\circledR}$ e Telegram ${ }^{\circledR}$. Já a quantidade de horas jogando teve correlação positiva com bate-papo, mas correlação negativa com editor de textos e apresentações, o que pode indicar que pessoas que jogam muito na amostra pesquisada, talvez o façam por dispositivos móveis, no qual é mais fácil utilizar bate-papos do que programas de edição.

A quantidade de horas jogando teve ainda correlação negativa com média geral e com horas semanais de trabalho, o que talvez revele que nesta amostra as pessoas que mais jogam são aquelas que apenas estudam. Como não houve correlação com horas de estudo semanal, acredita-se que as pessoas que jogam mais, estudam a mesma quantidade de horas semanais, mas obtém notas menores.

Por outro lado, a média geral teve correlação positiva com uso de editor de textos e de apresentações e também com investigação social. Neste ponto, pode-se arguir que alunos que fazem trabalhos escritos ou por seminários, normalmente com auxílio de softwares de apresentação, possuem maior nota. Além disso, a relação de média geral com investigação social pode se dar pelo aluno conhecer melhor os outros membros do grupo nos trabalhos ou até mesmo conhecer melhor o professor que irá avaliá-los, consultando sites de redes sociais, como o Facebook $^{\circledR}$, Twitter $^{\circledR}$ ou LinkedIn ${ }^{\circledR}$, ou repositórios de currículos como a plataforma Lattes.

$\mathrm{O}$ uso de editor de texto teve correlação positiva com praticamente todos os outros usos, menos o acompanhamento do feed de amigos. Destaca-se a correlação com uso para apresentações $(0,62)$, o que pode indicá-lo como uma "porta de entrada" para outros usos de computador, assim como o uso de e-mail que teve alta correlação com todos os usos de computadores. Neste sentido, o comportamento de uso de computadores para tal amostra, pode indicar que provavelmente eles foram levados a utilizar editores de texto e de apresentações, devido a atividades universitárias, e, a partir daí, começaram a familiarizar-se com outros tipos de software.

Já o uso de sistemas de gestão teve alta correlação com planilhas eletrônicas $(0,46)$, revelando que são usos bastante similares e voltados para atividades profissionais específicas.

Bate-papo teve alta correlação com acompanhar feed dos amigos, que por sua vez tem alta correlação com investigação social, demonstrando uma certa unidade em relação ao uso de redes sociais.

Com relação às escolhas dos alunos, observa-se que a quantidade de horas no smartphone teve correlação positiva com gosto e aptidão pela profissão. Como essa relação pode ter dois sentidos, argui-se que pessoas mais engajadas na futura carreira aproveitem as potencialidades atuais dos smartphones para desenvolver suas redes de contatos ou utilizar aplicativos que os ajudem em suas atividades.

Sobre a classificação do curso pelos alunos, foi demonstrada correlação positiva com o uso de computadores para interação com portais de comunicação, assim como importância do nível dos professores e atividades de ativismos na internet. Tais relações parecem demonstrar que cursos com um maior número de ações realizadas por professores obtém maior visibilidade na Internet e isso impacta na forma como os alunos avaliam sua qualidade.

Além disso, foi observada correlação positiva entre acompanhar o feed de amigos e dar importância a conteúdo 
prático, assim como dar importância para infraestrutura da universidade e usar computador para tratamento de imagens. Tais relações parecem seguir uma lógica similar ao apontado anteriormente, no qual a publicidade de atividades práticas realizadas por alunos nas redes sociais tende a fazer seus contatos valorizarem tais atividades assim como valorizar a infraestrutura da instituição.

Sobre as escolhas futuras dos alunos observa-se correlação entre acompanhar empresas na internet com escolher curso pelo gosto da profissão ou por perceber oportunidades de emprego ou por vontade dos familiares. Tal fato pode ser explicado por conta da forte atuação das empresas na Internet e nas redes sociais acabar influenciando alunos da área que desejam inserção no mercado de trabalho, ao passo que conhecem a profissão.

Ainda sobre este tema, observa-se correlação entre uso de sistemas de gestão e escolha do curso para favorecer emprego, o que pode indicar, neste caso, um perfil de universitários que trabalham desde o primeiro período.

No que tange aos jogos, nota-se que o tempo (em anos) usando computadores regularmente teve correlação positiva com jogos de RPG, MMORPG, Ação e Tiro, o que provavelmente se deve a esse tipo de jogos serem os precursores em computadores.

O nível de renda e as horas de trabalho semanais não tiveram correlação com nenhum tipo de jogo, mas tempo de estudo semanal apresentou correlação positiva com jogos de Quiz, o que pode ser uma indicação de que esses tipos de jogos são utilizados como reforços nos estudos, mesmo que de forma não planejada.

A quantidade de horas semanais no smartphone teve relação apenas com jogos Arcade, enquanto que as horas semanais no computador tiveram correlação positivas com jogos de RPG, MMORPG, Ações, Esportes, Arcade e Tiro. Tais relações se devem aos jogos melhor suportados por cada tipo de dispositivo.

Embora fosse esperado o contrário, a média geral teve correlação negativa com jogos de RPG, MMORPG, Esportes e Tiro, o que talvez sofra influência da quantidade de horas jogadas, mas não do tipo de jogo em si.

As atividades no computador de ativismo, acompanhamento de empresas, edição de imagens, uso de sistemas de gestão e interação com portais de comunicação tiveram correlação positiva com todos os tipos de jogos. Tais atividades se mostram interessantes, pois refletem aspectos importantes de jogadores, uma vez que estão sempre atentos a novidades de empresas de jogos, seja diretamente pelo site da empresa ou por portais de comunicação, além de serem razoavelmente organizados para cobrar melhorias.

O uso do computador para acompanhar notícias e preparar apresentações teve correlação positiva com jogos de Quiz, o que se mostra coerente pois são atividades que se ajudam mutuamente.

Ao se analisar a autoavaliação realizada pelos entrevistados, nota-se que jogos de Quiz teve correlação positiva com a autoavaliação de boas relações, o que pode indicar melhor índice de conhecimentos gerais.

Jogos de RPG e MMORPG tiveram correlação positiva com autoavaliação de inteligência, mas correlação negativa com competência e realização profissional, o que pode indicar que embora tais jogos proporcionem conhecimentos e análises diferenciadas, os entrevistados parecem ter dificuldade de transpor tais conhecimentos para atividades profissionais. Tal argumento pode ser reforçado pelo fato de pessoas que praticam jogos de RPG e MMORPG terem correlação positiva com a escolha do curso ter sido feita por vontade dos familiares.

Ainda sobre a análise de autoavaliação, o tempo usando computador (em anos) teve correlação positiva com auto avaliação de inteligência e de habilidade em falar em público, enquanto que as horas semanais usando computador tiveram correlação negativa com realização profissional e felicidade, mas positiva com inteligência. Aqui novamente se ressalta a possível dificuldade de transpor os conhecimentos trabalhados com as atividades profissionais que serão desenvolvidas.

Já a quantidade de horas no smartphone teve correlação positiva com liderança ao passo que a quantidade de horas jogando teve relação positiva com inteligência, mas negativa com lidar com pessoas, felicidade e competência profissional.

\subsubsection{Análise de ANOVA}

Nesta seção, as análises são focadas nos usos de computador, procurando revelar possíveis diferenças a partir de grupos específicos.

Ao se analisar o sexo dos entrevistados, nota-se um uso maior de editores de texto e formatadores de apresentação pelas mulheres. Já a renda, novamente não demonstra qualquer tipo de diferença estatisticamente significativa, muito embora a ocupação revele diferenças no que tange a interação com portais de comunicação, uso de sistemas de gestão e uso de planilha eletrônica. No entanto, ao se analisar os pares, são demonstradas diferenças apenas marginalmente significativas entre entrevistados que apenas estudam e entrevistados que 
estudam e tem empresa.

Já em relação à área do curso, observara-se diferenças com relação ao uso para editores de texto, planilhas, apresentações, sistemas de gestão e ativismo. No que tange a ativismo, tal diferença se mostra entre estudantes de ciências humanas e estudantes da área de linguística. $\mathrm{O}$ uso de computadores para apresentações mostrou diferenças entre estudantes de engenharias e estudantes de linguística. $\mathrm{O}$ uso de editores de texto, mostrou diferenças entre estudantes de ciências agrárias com estudantes de ciências biológicas

No que tange à natureza da instituição, foi possível observar apenas diferenças significativas no uso de computadores para acompanhar/entrar em contato com empresas e para interagir com portais de comunicação, revelando uma maior importância deste uso nas instituições privadas.

Já em relação à intenção de atividade quando terminar o curso, nota-se uma diferença significativa no uso de Planilhas Eletrônicas, apresentações e ao entrar em contato com empresas, mas não sendo possível observar diferenças entre pares.

Com relação a jogos, foi observado que as mulheres tendem a gostar mais de jogos de Quiz, enquanto que os homens tendem a gostar mais de jogos de RPG, MMORPG, ação, esportes e tiro.

MMORPGs tem diferenças estatísticas entre estudantes de linguística e sociais aplicadas e entre estudantes de engenharias com biológicas, enquanto que os jogos de RPG só demonstraram diferenças entre estudantes de linguística e multidisciplinares. Este fato é relativamente interessante, pois destaca a área de linguística e artes como propícia para jogos de interpretação de papéis.

Jogos de simulação revelaram diferenças entre estudantes de linguística e engenharias, assim como entre estudantes de ciências da saúde e ciências biológicas. Já jogos de tabuleiro demonstraram diferenças entre estudantes de ciências sociais aplicadas e saúde. Estas comparações ressaltam como cursos nos quais a prática é relativamente arriscada, os jogos de simulação são bem procurados.

Ao comparar universidades públicas e privadas, nota-se que os alunos desta última tendem a dar maior importância para jogos de simulação, RPG, MMORPG, ação, esportes, tiro, arcade e puzzle, demonstrando um interesse variado por jogos.

Por fim, ao se analisar as decisões ao terminar a carreira, as pessoas que querem seguir carreira pública tiveram médias distintas de pessoas que desejam abrir o próprio negócio nos jogos de arcade e Quiz, o que pode estar relacionado ao citado acima, já que jogos desse tipo auxiliam nos estudos, especialmente em assuntos mais taxonômicos como ocorre em concursos públicos.

\section{Conclusões}

Este estudo teve como intenção analisar as relações entre uso de tecnologias de informação e comunicação e jogos nas escolhas e no desempenho dos estudantes de cursos de graduação de universidades da região Nordeste do Brasil, uma vez que a teoria da mediação cognitiva ressalta melhorias de desempenho e de empreendedorismo em estudantes com alto grau de uso de tecnologias de informação.

Com relação aos jogos cabe destacar os do tipo Quiz, uma vez que mostraram correlação tanto com horas semanais de estudo como também os alunos interessados em prestar concursos públicos e as mulheres.

Outro ponto interessante foram os jogos de Role Playing Game e Massive Multiplayer Online Role Playing Game terem uma correlação negativa com a nota da média geral, embora os alunos se considerem mais inteligentes. Adicionalmente, nota-se que tais alunos não se veem satisfeitos com sua carreira, tendo-a escolhido no geral por vontade dos familiares.

Ademais, todos os tipos de jogos demonstraram correlação com o uso de computadores para ativismo e para acompanhamento de empresas, o que pode indicar que jogadores no geral tendem a se organizar em comunidades e discutir melhorias para o grupo que fazem parte, ao passo que ficam atentos às ações realizadas pelas empresas. Embora tal uso provavelmente se inicie com empresas de jogos, pode ser um começo para desenvolvimento em ações futuras com outros tipos de organizações.

Além disso, alunos de universidades privadas tendem a usar mais o computador para entrar em contato com empresas e portais de comunicação no geral, quando comparados com alunos de universidades públicas.

Curiosamente o uso de e-mail e de editores de texto demonstrou correlação com todos os usos de computadores, o que os revela com uma porta de entrada para outros usos.

Por último, vale destacar que alunos que acompanham empresas pela Internet parecem ser impactados por essas 
informações para a escolha do curso, especialmente por conhecerem mais sobre a profissão e oferta de empregos. Tal ponto afeta também os familiares dos alunos, o que faz com que os mesmos tentem influenciar o aluno na escolha de determinados cursos.

Portanto, nota-se a importância de se observar as diversas relações dos alunos de graduação com o uso de tecnologias de informação, especialmente em busca de entender esse relacionamento, assim como pensar em formas de engajar os alunos nas atividades curriculares.

\section{REFERÊNCIAS}

BANDURA, A. Exercise of human agency through collective efficacy 12. Current Directions in Psychological Science, v. 9, n. 3, p. 75-78, 2000.

BITTENCOURT, João Ricardo; GIRAFFA, Lucia Maria. Role-playing games, educação e jogos computadorizados na cibercultura. I Simpósio de RPG em Educação, p. 14, 2003.

CARNEIRO, R. E.; GALlE, B. A.; LIMA NETO, F. B.; SILVEIRA, D. S. Sistema Hipermídia Adaptativo Educacional Baseado em Estilos de Aprendizagem. Simpósio Brasileiro de Informática na Educação, v. 24, n. 1, p. 993, 2013.

COOPER, D. R.; SCHINDLER, P. S. Métodos de pesquisa em administração. Porto Alegre: Bookman, 2011.

DUCHENEAUT, Nicolas; MOORE, Robert J. - More Than Just 'XP': learning social skills in massively multiplayer online games. Interactive Technology e Smart Education, v.2, n. 2, p. 89-100, 2005.

HAIR JR, J. E.; BLACK, W. C. BABIN, B. J. ANDERSON, R. E. TATHAM, R. L. Análise Multivariada de Dados. 6 ed. Porto Alegre: Bookman, 2009.

HEROLD, Benjamin. Researchers see video games as testing, learning tools. Education Week, v. 32, n. 37, p. 14-15, 2013.

HEROLD, Benjamin. Technology in education: An overview. Education Week, v. 20, p. 129-141, 2016.

LUSZCZYNSKA, A.; SCHWARZER, R. Social Cognitive Theory. In: Predicting health behaviour. McGrawHill Education (UK), 2005.

NASCIMENTO, A. M.; LUFT, M. C. M. S.; SILVEIRA, D. S. Muito mais do que contato com os amigos: Possibilidades de uso de uma rede social virtual. In: Anais... XXXVIII Encontro Nacional da ANPAD. Rio de Janeiro, 2014.

PEREIRA, Ariane Rodrigues; DALMAU, Marcos Baptista Lopez. As competências virtuais individuais desenvolvidas pela graduação a distância em um curso de administração: o caso da Universidade Federal de Santa Catarina. Revista Gestão Universitária na América Latina-GUAL, v. 8, n. 2, p. 90-112, 2015.

PRAZERES, Michelle. Empresa HD, aluno monitor: a Microsoft e a construção da crença nas tecnologias. Educação e Pesquisa, v. 41, n. 2, p. 527-542, 2015.

RANDI, Marco Antonio Ferreira; CARVALHO, Hernandes Faustino de. Learning through role-playing games: an approach for active learning and teaching. Revista Brasileira de Educação Médica, v. 37, n. 1, p. 80-88, 2013.

SCHUNK, Dale H. Learning theories. Printice Hall Inc., New Jersey, p. 1-576, 1996.

SEPÉ, Cláudia Presser. Usuários articulados em torno de MMORPGs: o chat como elemento marcador da posição socializante e identitária do sujeito jogador. Universidade do Vale do Rio dos Sinos. Acesso às 17 h25 em, v. 4, 2005.

SELWYN, Neil. Education and technology: Key issues and debates. Bloomsbury Publishing, 2016.

SILVA, Leonardo Xavier de Lima e. Processos Cognitivos em Jogos de Role-Playing: World of Warcraft vs. Dungeons e Dragons. Dissertação (Mestrado em Psicologia), Programa de Pós-graduação em Psicologia Cognitiva, Universidade Federal de Pernambuco, Recife, 2008.

STEINKUEHLER, Constance; CHMIEL, Marjee. Fostering scientific habits of mind in the context of online play. In: Proceedings of the 7th international conference on Learning sciences. International Society of the Learning Sciences, 2006. p. 723-729. 
Desempenho Acadêmico e Uso de Tecnologias de Informação e Comunicação: Uma Análise com Estudantes de Graduação do Nordeste Brasileiro

Revista Gestão.Org, v. 15, Edição Especial, 2017. p. 138-146

ISSN 1679-1827

http://www.revista.ufpe.br/gestaoorg

VAFA, Shahrzad; CHICO, Diane E. A needs assessment for mobile technology use in medical education. International Journal of Medical Education, v. 4, n. 1, p. 230-235, 2013. 